\title{
Active Power Sharing and Frequency Restoration in an Autonomous Networked Microgrid
}

\author{
Mohsen Eskandari, Li Li, Mohammad Hassan Moradi, Pierluigi Siano, Senior Member, IEEE, and \\ Frede Blaabjerg, Fellow, IEEE
}

\begin{abstract}
Microgrid (MG) concept is considered as the best solution for future power systems, which are expected to receive a considerable amount of power through renewable energy resources and distributed generation units. Droop control systems are widely adopted in conventional power systems and recently in MGs for power sharing among generation units. However, droop control causes frequency fluctuations, which leads to poor power quality. This paper deals with frequency fluctuation and stability concerns of $f-P$ droop control loop in MGs. Inspired from conventional synchronous generators, virtual damping is proposed to diminish frequency fluctuation in MGs. Then, it is demonstrated that the conventional frequency restoration method inserts an offset to the phase angle, which is in conflict with accurate power sharing. To this end, a novel control method, based on phase angle feedback, is proposed for frequency restoration in conjunction with a novel method for adaptively tuning the feedback gains to preserve precise active power sharing. Nonlinear stability analysis is conducted by drawing the phase variations of the nonlinear secondorder equation of the $\delta$-P droop loop and it is proved that the proposed method improves the stability margin of $f-P$ control loop. Simulation results demonstrate the effectiveness of the proposed method.
\end{abstract}

Index Terms - Distributed generation (DG), Droop control, Frequency restoration, Microgrid (MG), Power control, Renewable energy resources (RESs).

\section{INTRODUCTION}

$M^{2}$ ICROGRID (MG) concept has been introduced to deal with economical and technical concerns related to the integration of renewable energy sources (RESs) and distributed generation (DG) units into conventional power systems [1]. MG is managed by an energy management system for achieving economic efficiency as well as controlling DG units and controllable loads [2]-[3]. The control systems implement power sharing and voltage

M. Eskandari and L. Li are with the School of Electrical and Data Engineering, University of Technology Sydney, Sydney, NSW, 2007 Australia (e-mail: mohsen.eskandari@uts.edu.au; 1 i.li@uts.edu.au).

M. H. Moradi is with the Department of Electrical Engineering, Bu-Ali Sina University, Hamedan 65168 63611, Iran (e-mail: mh_moradi@yahoo.co.uk). regulation while securing dynamic stability of the MG [4]. Droop control method, which works well in conventional power systems, is considered for power sharing in MGs. The frequency-active power $(f-P)$ droop control loop [5] is adopted to distribute the demanded power among DG units according to their capacity. The voltage magnitude-reactive power $(V-Q)$ droop loop is developed for voltage regulation to prevent circulating current among DG units. Droop control is a simple, effective and decentralized method that does not depend on communication network, which makes it a cost-effective and reliable control system [6]-[7]. Nevertheless, there are some major challenges related to the droop control method in power sharing that are listed as follows:

1) cross-coupling of $f-P$ and $V$ - $Q$ droop loops because of the droop control performance dependency on power network impedance;

2) insufficiency of the droop control for achieving accurate reactive power sharing;

3) voltage deviation from the nominal band;

4) frequency deviation from the nominal value;

5) high sensitivity of the dynamic performance to the droop gains, and narrow stability margins.

The low $\mathrm{X} / \mathrm{R}$ ratio of the interconnecting power lines (feeders) in MGs, located at low-voltage grids, interrelates $f-P$ and $V-Q$ control loops, which are decoupled by employing physical or virtual impedances [8]. The second and third challenges are related to the $V$ - $Q$ droop control loop. Voltage drop over the feeder impedance causes inaccuracy in reactive power sharing which, in turn, causes circulating current among DG units [9]-[18]. The proposed methods in [9]-[12] used in parallel MGs are mostly based on injecting some signal or on the computation and estimation of the MG main bus voltage and voltage drop through the feeders to accordingly modify the droop controllers. The recent works cope with the issue in networked MGs by adopting the communication-based distributed control [14]-[18]. The power quality concerns related to voltage deviation are addressed by proposing a method at the secondary level [19]-[20].

The fourth and fifth challenges, which are considered in

P. Siano is with the Department of Management and Innovation Systems, University of Salerno, Via Giovanni Paolo II, 132, Fisciano (SA) - 84084 Italy (e-mail: psiano@unisa.it).

F. Blaabjerg is with the Department of Energy Technology, Aalborg University, 9220 Aalborg East, Denmark (e-mail: fbl@et.aau.dk). 
this paper, are related to the $f-P$ control loop. Despite of appropriate operation of the droop control in active power sharing, the frequency generally deviates from its nominal value. In addition, droop control performance depends on droop gains. A large droop gain helps achieve accurate and fast power sharing, while it makes the frequency deviation more and the stability margin narrower [21]. Adaptive droop control is proposed in [22] to conserve the stability by adding derivative terms of $P$ and $Q$ to the conventional $f-P$ and $V$ - $Q$ droop control loops. However, the derivative term intensifies the noises and imposes impulses to the system. Arctan $f$-P droop is proposed in [23] to modify the droop gain in various load conditions and to improve the dynamic stability, but it may be in conflict with power sharing in severe load conditions.

In [24], the synchronverters concept is proposed for power converter-based MGs to mimic the drooping mechanisms (swing equation) in synchronous generators, and the stability is improved by providing a virtual inertia. However, the swing equation must be solved by an iterative method to calculate the operating frequency, which makes the control system more complicated and less effective in comparison to the traditional one. A phase angle-active power $(\delta-P)$ control loop is proposed to bound the frequency deviation [25]. In [26], a supplementary control loop is added to the $\delta$ - $P$ control loop to improve the dynamic stability. However, the angle droop control frequency needs GPS-based timing signals to synchronize different controllers. Besides, frequency deviation is still a matter of concern. In [27] a $V$-I droop control is proposed for fast dynamic performance and a GPS signal is used for synchronization with fixed frequency. However, lack of inertia of the $V$ - $I$ control system is a matter of dynamic stability concern, especially in networked MGs, and achieving the accurate power sharing is a challenging issue as the voltage is not a global variable.

The conventional $f-P$ droop control is the most straightforward and popular method for power sharing in MGs and scholars try to address the frequency deviation and stability issues. The control methods which are proposed for frequency restoration are included in three main categories: centralized [28], [29], decentralized [30]-[31] and recently distributed consensus control systems [32]-[38]. In almost all methods proposed in the literature for frequency restoration, the frequency deviation from the nominal value is used to generate the corresponding control signal via an integrator controller in different control structures. Frequency restoration in this way is however in conflict with power sharing. The $f-P$ droop loop dynamically controls the phase angle. This leads to different phase angles at individual droop controllers and accurate power sharing as a result (the sensitivity of the active power to the phase angle is high, proportional to the square of voltage magnitude). On the other hand, the integral term of frequency variation, which is used as a control signal to modify the operating frequency, inserts an offset to the phase angle of the output power after each disturbance. This causes considerable inaccuracy in power sharing even due to limited load-changing steps. Although this conflict has been mentioned in only few works [31], [32], [36], the proposed methods are complex and not effective. The reason is that the issue is not explored appropriately and clearly which has led to some non-promising solutions which also raise the stability concern in networked MGs. In [31] Wavelet transform, a mathematical function for change detection, is adopted to detect load changes. Then, after reaching the steady state, the frequency restoration loop is activated to eliminate the communication link requirement. However, frequency restoration loops at all DG units must be coordinated and be activated at a same time due to different frequency dynamics at individual loads, so communication link is needed, otherwise the issue still exists. The proposed method also imposes delay to the frequency restoration process which is not desirable in MGs with frequent and random load changes. The method proposed in [32] works based on a complex consensus protocol which requires a high band-width communication network and also affects the dynamic stability of networked MGs. The authors in [36] developed a method which also needs a communication link to measure all demanded active powers in an MG and then compute and use the contribution of each DG unit to support the active power. Then, the error related to the active power due to the frequency restoration process is removed through an integrator. The proposed method influences the simplicity and decentralized feature of the droop controller due to its complexity which needs time and efforts to be implemented.

This issue is investigated in this work clearly and consequently a novel control scheme for frequency restoration and stability improvement in droop-based MGs is developed. Firstly, a new analogy between a droop control in conventional power systems and a droop control in MGs is presented. Then, inspired from the synchronous generator, virtual damping is proposed to improve the dynamic performance and to suppress frequency oscillations in MGs followed by corresponding hints to adjust virtual damping to be effective and consistent with power sharing. The proposed virtual damping significantly helps the frequency restoration loop to be simple and effective. Then an important and critical issue with droop control and frequency restoration in networked MGs is highlighted, which has not been acknowledged in majority of works, followed by proposing a proper method. In the developed method, the phase angle is adopted as state feedback to improve the stability margin of the conventional droop controller. The feedback signal not only secures the dynamic stability of the $f-P$ droop loop, but also restores the frequency deviation. Moreover, the feedback gain is adaptively tuned to secure accurate active power sharing. In the proposed method, the secondary controller, i.e. frequency restoration loop, works tightly with the primary controller, i.e. the modified droop controller, so that the overall time constant is equal to or even lower than that of the conventional droop loop without taking the secondary loop into consideration. Finally, nonlinear stability analysis is proposed to prove the stability of the proposed method in a variety of operating points.

The contributions of the proposed method are listed as 
follows:

- virtual damping is proposed and designed to damp the low frequency oscillations of active power and to suppress frequency fluctuations in droop-controlled MGs;

- the issue related to the conventional frequency restoration loop is investigated and it is clarified that frequency restoration leads to inaccuracy in active power sharing, which is not desirable regarding the small scale of DG units and current limit of power converters that may put MGs exposed to collapse;

- a novel method for immediate frequency restoration coordinated with droop controllers in power sharing is proposed, which is vital to avoid frequency instability regarding the frequent and random load changes in MGs. The idea of slack bus in bulk power systems, is inspired and adapted for MGs to preserve frequency stability;

- the proposed method is fast, simple and effective to preserve plug-and-play capability and to secure dynamic stability of droop-controlled MGs.

In the next section, MGs architecture and conventional droop control system are described. The modified droop controller is presented in Section III. The proposed method for frequency restoration is developed in Section IV. Nonlinear stability analysis is conducted in Section $\mathrm{V}$ followed by simulation results which are presented in Section VI to demonstrate the effectiveness of the proposed scheme. Section VII presents a discussion, and finally, conclusions are drawn in Section VIII.

\section{MG DESIGN}

\section{A. MG Architecture}

The considered microgrid topology is depicted in Fig. 1. Voltage source inverters (VSIs) (grid forming inverters) represent generation units, since RESs and DG units are mostly connected to the grid via power converter interfaces. Furthermore, the main duty of the MG control system, i.e. power sharing and voltage regulation, is accomplished by controlling VSI converters. Non-dispatchable RESs like solar cells or wind turbines, which normally operate at maximum power point tracking, are connected to the grid through current source inverters (CSIs) (grid feeding inverters) [4].

The MG is connected to the main grid at the point of common coupling (PCC) that is the MG's main bus by which MG's common loads are supplied. The power network might be in parallel, series-parallel or meshed forms depending on the DG unit's locations as well as reliability, efficiency and power quality purposes. MG central controller (MGCC) implements the energy management, as well as operating mode treatment (islanded, connected, transition and synchronization) in coordination with the upstream grid. The MGCC sends optimal set-points as well as nominal values to DG units through low bandwidth communication link (LBWCL).

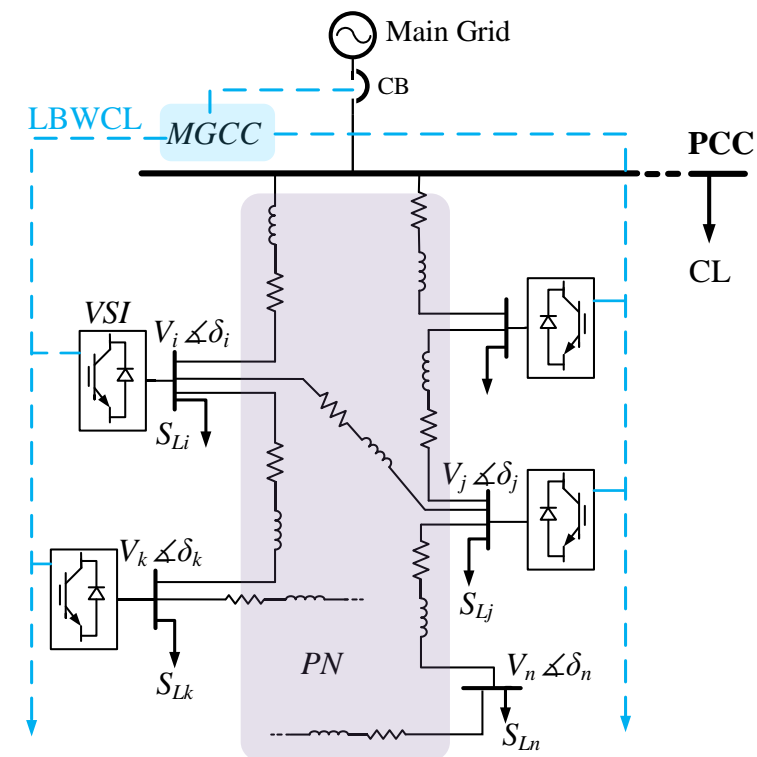

Fig. 1. MG topology (PN: Power Network; CL: Common Load; CB: Circuit Breaker).

\section{B. Droop Control in MGs}

A droop-like control system, which mimics the behavior of conventional synchronous generators, is adopted for power sharing in islanded MGs. The instantaneous active and reactive power flowing through the $i j^{\text {th }}$ interconnecting power line $(P$ and $Q)$ are obtained as:

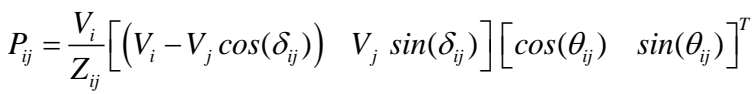

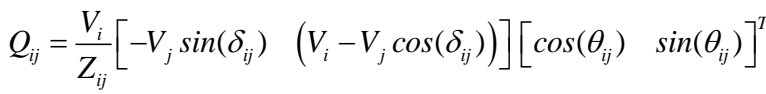

where $i \& j$ indices denote the $i^{t h}$ and $j^{\text {th }}$ nodes, $V$ and $\delta$ are the magnitude and phase angle of the voltage at end nodes, respectively, and $Z$ and $\theta$ are amplitude and phase angle of the feeder impedance. For inductive power networks with high $\mathrm{X} / \mathrm{R}$ ratio $\left(\theta \approx 90^{\circ}\right), P$ and $Q$ are given as:

$$
\begin{aligned}
& P_{i j}=\frac{V_{i} V_{j} \sin \left(\delta_{i j}\right)}{X_{i j}} \\
& Q_{i j}=\frac{V_{i}}{X_{i j}}\left(V_{i}-V_{j} \cos \left(\delta_{i j}\right)\right)
\end{aligned}
$$

Because of the high sensitivity of active power to phase angle in inductive power networks, active power is regulated by controlling the frequency (which dynamically controls the phase angle). A similar scenario is executed for reactive power control by controlling the voltage magnitude. The sensitivity of reactive power to the voltage magnitude is not as high as the sensitivity of active power to the phase angle. Although MGs are located in lowvoltage grids in which the $\mathrm{X} / \mathrm{R}$ ratio of the interconnecting cable impedance is not too high, the overall $\mathrm{X} / \mathrm{R}$ ratio of the grid is high enough because of installing LCL filters, isolating transformers and virtual inductance. The droop equations for DG $i$ are given as: 


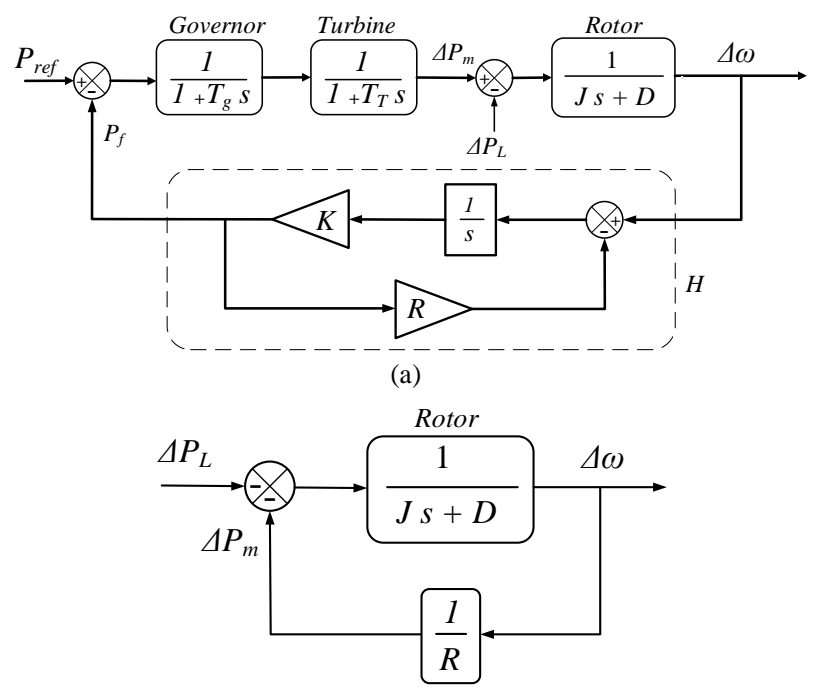

(b)

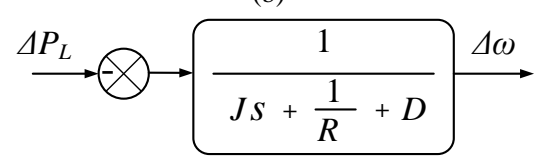

(c)

Fig. 2. Block diagram of droop control in conventional power systems: (a) frequency control with droop loop; (b) the simplified droop loop; (c) equivalent transfer function.

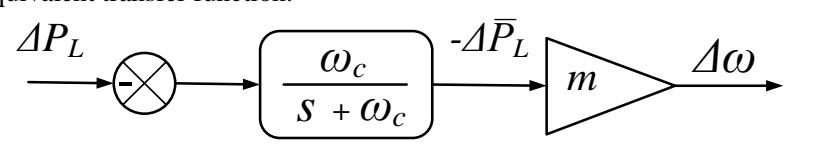

(a)

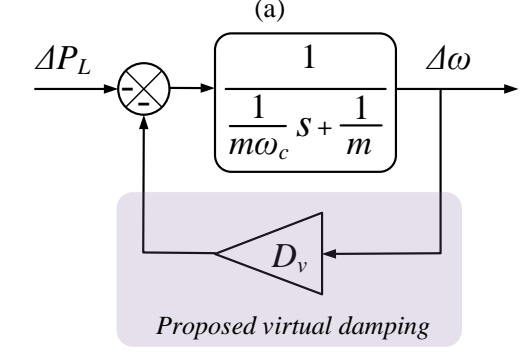

(b)

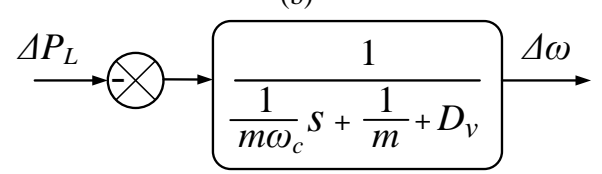

(c)

Fig. 3. Block diagram of droop control in in MG: (a) the $f-P$ loop; (b) the proposed virtual damping; (c) equivalent transfer function.

$$
\begin{aligned}
& \omega_{i}=\omega_{0}-m_{i} \bar{P}_{i} \quad(\Delta \omega=-m \bar{P}) \\
& V_{i}=V_{0}-n_{i} \bar{Q}_{i}
\end{aligned}
$$

where $\omega\left(\omega_{0}\right), V\left(V_{0}\right)$ are the operating (nominal) radian frequency and voltage magnitude, respectively, $m \& n$ are the droop gains and $\bar{P} \& \bar{Q}$ are the average output active and reactive power obtained by passing instantaneous $P$ and $Q$ through a low-pass filter $\left(\omega_{c} /\left(s+\omega_{c}\right)\right)$. Power sharing is implemented by assigning droop gains to DG units so that the following equilibriums are obtained:

$$
\begin{aligned}
& m_{1} \bar{P}_{1}=\ldots=m_{i} \bar{P}_{i}=\ldots=m_{z} \bar{P}_{z} \\
& n_{1} \bar{Q}_{1}=\ldots=n_{i} \bar{Q}_{i}=\ldots=n_{z} \bar{Q}_{z}
\end{aligned}
$$

where $z$ is the number of dispatchable DG units. As the frequency is a global variable throughout the MG, active power sharing is implemented appropriately according to the droop gains. However, the frequency deviates from its nominal value when a load change happens in the system as a disturbance. This leads to frequency fluctuation after some disturbance and thus the poor power quality.

\section{MODIFIED DROOP CONTROLLER}

\section{A. Virtual Damping and Design Process}

The droop control method is inspired from the behavior of synchronous generators in the bulk power systems where increasing output power leads to frequency (rotor speed) drop. The block diagram of the droop control implemented in conventional power systems is shown in Fig. 2(a). The feedback loop is in charge of frequency restoration. In lieu of restoring the frequency to the nominal value, it is dropped with specified slope which is implemented by assigning a droop gain. The transfer function of the feedback loop $H$, from $\Delta \omega$ to $P_{f}$ (feedback signal), is obtained as:

$$
H(s)=\frac{1 / R}{(1 / R K) s+1}
$$

where $R$ is the droop gain, and $K$ is the integrator gain. The time constants of governor and turbine are large in comparison to the rotor time constant, so that they can be considered constant during our study on the rotor dynamic. Furthermore, the gain $K$ is considered a big value to make the time constant of the feedback loop small $(H \approx 1 / R)$, and thus the simpler model in Fig. 2(b) is obtained.

The equivalent droop control transfer function of synchronous generators is given as in (10) which is depicted in Fig. 2(c):

$$
\frac{\Delta \omega}{\Delta P_{L}}=\frac{-1}{J_{S}+D+1 / R}
$$

where $J$ is the moment of inertia of rotating elements, $D$ is the damping factor, and $\Delta P_{L}$ represents the load changes.

On the other hand, the block diagram of the $f-P$ droop control in MGs, presented in (5), is shown in Fig. 3(a), in which the multiplication of the average load power (variation) by the droop gain specifies the operating frequency (variation) of the system. The equivalent transfer function of the droop loop in MGs is given as in (11) which is shown in Fig. 3(b) as:

$$
\frac{\Delta \omega}{\Delta P_{L}}=\frac{-1}{\left(1 / m \omega_{c}\right) s+1 / m}
$$

Associating (10) and (11) reveals that:

- the moment of inertia of the rotating element restores the energy in the rotor mass and provides fast response to disturbances. This phenomenon in turn, provides plenty of moment for the control system to be effective and stable. On the other hand, because of lack of rotating element (inertia) in the converter-based MGs and, on top of that, fast response of power converters, the low-pass filter could be regarded as a virtual inertia $J_{v}=1 / m \omega_{c}$, which inserts a time constant in the droop controller, $T_{d}$ $=1 / \omega_{c}$. In this way, the cut-off frequency of the low-pass filter influences the dynamic response of the droop controller; 
- the damping factor $(D)$ exists in synchronous generators because of the mechanical friction and electrical damping provided by the damper winding. The damping factor in conventional power systems suppresses oscillations and helps the generator to be in synchronism with the power grid. However, there is no damping source in the $f-P$ droop loop in MGs. So a virtual damping $\left(D_{v}\right)$ is proposed to diminish frequency fluctuations in MGs. The virtual damping loop can be implemented in the control system of DG units as shown in Fig. 3(b). The equivalent transfer function of the modified droop loop in MGs is given as:

$\frac{\Delta \omega}{\Delta P_{L}}=\frac{-1}{\left(1 / m \omega_{c}\right) s+D_{v}+1 / m}$

The following criteria should be considered in selecting the appropriate virtual damping factor in MGs:

1) the time constant of the modified droop control loop in MGs is updated as:

$$
T_{d}^{\prime}=\frac{1}{\omega_{c}+m \omega_{c} D_{v}}
$$

So $D_{v}$ should be large enough to be effective as the droop gain $(m)$ is a small value;

2) the updated droop gain is given as:

$$
m_{i}^{\prime}=\frac{m_{i}}{1+m_{i} D_{v i}}
$$

So $D_{v}$ should not be too large to make the effective droop gain so small;

$3)$ the power sharing still needs to be established according to (7). Substituting (14) into (7) results:

$$
\frac{m_{1}}{1+m_{1} D_{v 1}} \bar{P}_{1}=\ldots=\frac{m_{i}}{1+m_{i} D_{v i}} \bar{P}_{i}=\ldots=\frac{m_{z}}{1+m_{z} D_{v z}} \bar{P}_{z}
$$

So the following criteria must be considered in selecting the virtual damping factor:

$$
m_{1} D_{v 1}=\ldots=m_{i} D_{v i}=\ldots=m_{z} D_{v z}
$$

As shown in (13), the virtual damping makes the time constant smaller (faster response), while (14) reveals that adopting a virtual damping factor, the effective droop gain is smaller than the original one (indicating an improved stability margin). Equation (16) secures the accuracy in power sharing. So it is suggested to select $D_{v}$ so that $m D_{v}=1$. Overall, the inserted virtual damping factor, not only reduces the frequency fluctuation in the MG, but also improves the dynamic stability as it makes the actual droop gain smaller without distorting power sharing accuracy and pace. Still, an appropriate control strategy is required to restore the frequency deviation.

\section{B. Feedforward loop}

In addition to the virtual damping factor described earlier, the droop control loop is further modified by adding a feedforward loop to improve the dynamic response. The feedforward loop is an upgraded derivative controller used to remove ripples caused from pure derivative term as:

$$
\frac{T s}{1+\gamma T s}
$$

where $T$ is the feedforward gain and $\gamma$ is a small value $(0.1)$ [39].

\section{FREQUENCY RESTORATION}

This section proposes an adaptive control technique based on a phase angle (delta) feedback loop, to achieve frequency restoration, which is also consistent with power sharing. The schematic block diagram of the upgraded $f-P$ droop control loop for active power sharing as well as the proposed frequency restoration loop is illustrated in Fig. 4.

\section{A. Issues Related to the Conventional Frequency Restoration Loop}

The $f-P$ droop loop is reconstructed as in (18), noting that the virtual damping and feedforward loops are ignored to investigate the frequency restoration loop:

$$
\omega=\omega_{0}-m \bar{P}-\Omega
$$

where $\Omega$ is the control signal coming from the frequency restoration loop given as:

$$
\dot{\Omega}=k\left(\omega-\omega_{0}\right)
$$

where $k$ is the controller gain. The relation between the frequency and the phase angle variation is obtained as:

$$
\dot{\delta}=\omega-\omega_{0}
$$

So (18) is updated as:

$$
\omega=\omega_{0}-m \bar{P}-k \delta
$$

In this way, the phase angle $(\delta)$ feedback loop is added to the conventional droop control. Equation (21) works appropriately for frequency restoration, since the frequency is a common variable at all MG nodes. However, rigorous scrutiny reveals a drawback of the method for constant $k$. Equation (21) is developed for $i^{\text {th }}$ and $j^{\text {th }}$ controllers as:

$$
\begin{aligned}
& \omega_{i}=\omega_{0}-m_{i} \bar{P}_{i}-k_{i} \delta_{i} \\
& \omega_{j}=\omega_{0}-m_{j} \bar{P}_{j}-k_{j} \delta_{j}
\end{aligned}
$$

Subtracting (22) from (23) results (noting that if accurate power sharing is established then $\left.m_{i} \bar{P}_{i}=m_{j} \bar{P}_{j}\right)$ :

$$
\omega_{i}-\omega_{j}=k_{j} \delta_{j}-k_{i} \delta_{i}
$$

In contrast to frequency, the phase angle is not identical at all generation nodes. This is because phase angle variation depends on the droop gains (DG unit capacity) and power network topology. So this means that $k_{i} \delta_{i} \neq k_{j} \delta_{j}$ for fixed $k$ 's, and based on (24) leads to $\omega_{i} \neq \omega_{j}$, which is not true in steady state. Therefore, (24) is not reachable, which means $m_{i} \bar{P}_{i} \neq m_{j} \bar{P}_{j}$. Justification is that the third term of (18) inserts an offset into the phase angle of the voltage at corresponding nodes which is not consistent with accurate power sharing. This offset is not big enough (for small $k$ ) to disturb power sharing immediately. But, this offset becomes large enough to disturb precise power sharing after a few disturbances, which is not desirable regarding the small scale of DG units and strict current limit of converters. Adopting large $k$ to achieve fast frequency restoration leads 
to more inaccuracy in power sharing.

To investigate this issue, let take the closed loop transfer function $C L_{t f}$ (from + to $P$ ) into consideration as (suppose that $k$ is a fixed value in Fig. 4):

$$
C L_{t f}=\frac{\left(A \omega_{c} T+A m \omega_{c} \gamma T\right) s+A m \omega_{c}}{\gamma T s^{3}+B s^{2}+C s+k_{i} \omega_{c}+A m \omega_{c}}
$$

where

$$
\begin{aligned}
A & =V_{i} V_{j} / X_{i j} \\
B & =1+\gamma T \omega_{c}+\gamma T k_{i}+T \omega_{c} D_{v}+m \gamma T \omega_{c} D_{v} \\
C & =\omega_{c}+A m \gamma T \omega_{c}+A T \omega_{c}+k_{i}+\gamma T k_{i} \omega_{c}+m \omega_{c} D_{v}
\end{aligned}
$$

The step response of the equivalent second-order transfer function is given in Fig. 5. It is concluded that although increasing control parameter $(k)$ improves the dynamic response of the $f-P$ control loop, this ends up with an error in the steady state.

\section{B. Adaptive Phase Angle Feedback Loop for Frequency Restoration}

For the active power sharing to be implemented accurately, the following prerequisite is essential:

$$
k_{1} \delta_{1}=\ldots=k_{i} \delta_{i}=\ldots=k_{z} \delta_{z}
$$

However, the phase angle dynamics make the control system designer unable to assign static control parameters to achieve the condition required as in (26). On the other hand, the phase angle dynamics at other generation nodes are not available to design a decentralized controller. As mentioned earlier, the phase angle dynamics at individual generation nodes depend on the various elements like power network topology, allocation of DG units as well as allocation of loads and changes in load demand.

To address this issue, it is helpful to refer to the slack bus in the bulk power systems. In order to regulate the frequency in conventional power systems, a large synchronous generator with zero droop gain is taken as slack bus. The generator at the slack bus does not participate in the power sharing but is responsible for providing the spinning reserve. It produces power as much as it is needed to hold the balance between generation and consumption and to keep the frequency locked to the nominal value. On the other hand, it is not possible to assign one inverter as the slack bus in autonomous MGs due to the small-scale of DG units and strict current limits of converters [40]-[42]. Besides, it is not beneficial to put one DG unit as spinning reserve in MGs since DG units must work on the optimum/available operating point. So all DG units are involved in supporting demanded power, power losses and frequency regulation in MGs. Nevertheless, the idea of slack bus can be adopted to achieve the control target, i.e. fulfilling the requisite given in (26), in MGs. To this end, it is proposed to choose a given VSI as the master/slack VSI and coordinate other VSIs with the master/slack one. To this end, the control parameter of the master VSI (VSI $j$ ) is fixed to a constant value and the following rule is employed to tune control parameter of other controllers adaptively:

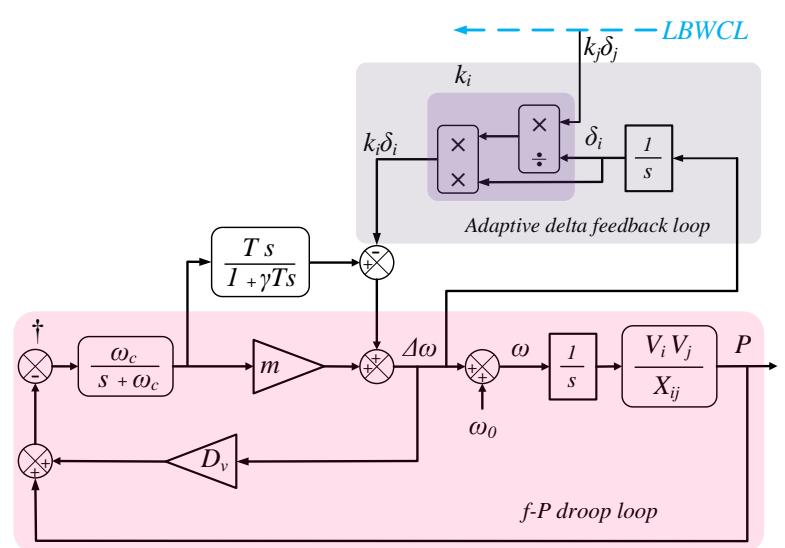

Fig. 4. Proposed adaptive feedback loop for frequency restoration.

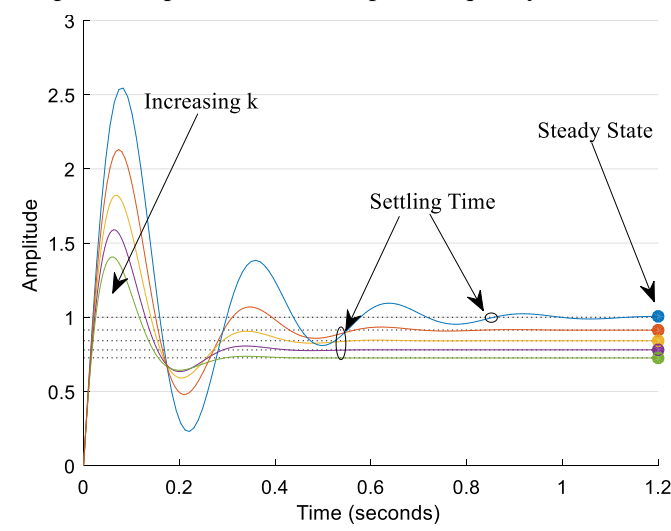

Fig. 5. Step response of the $f$ - $P$ droop loop.

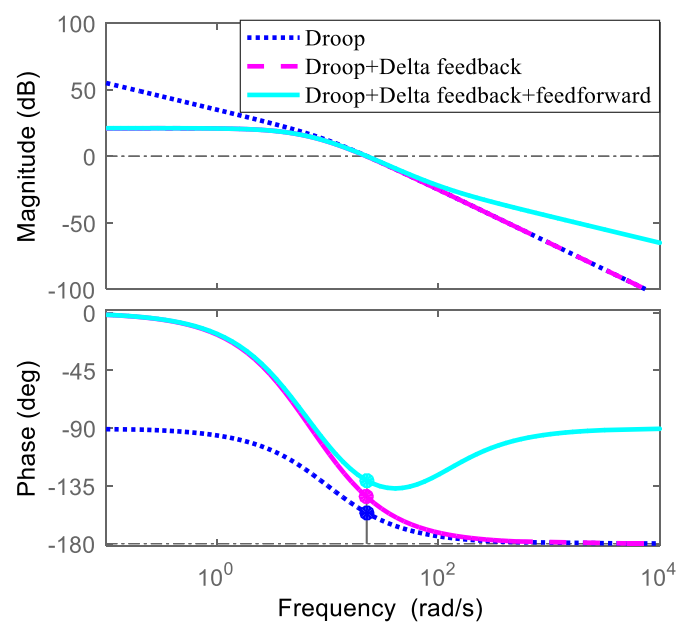

Fig. 6. Bode diagram showing improvement of the stability margin of control system by adopting delta feedback and feedforward terms.

$$
k_{i}=\frac{k_{j} \delta_{j}}{\delta_{i}}
$$

In contrast to the slack generator in power systems, the master/slack VSI in MGs participates in the power sharing (with non-zero droop gain), but with a fixed frequency restoration gain to coordinate other VSIs. Since the droop gain of master/slack VSI is not zero, it does not have to be a large DG unit.

In this way, the topology of the frequency regulation approach considering communication link is decentralized with centralized synchronization signal. Comparing to the distributed consensus control method [32], it is not necessary to exchange data (bidirectional) between adjacent 
controllers and only one-way data transfer (unidirectional) is enough to achieve the control goal, which reduces required bandwidth of the LBWCL. The other advantage of the proposed technique over consensus control is that consensus control imposes delay to the frequency restoration loop which is not desirable in MGs. Besides, consensus controller requires adding more integrators (poles) to the control system per each controller, which leads to a more complex control system with narrower stability margins. More importantly, the consensus protocol for frequency restoration is more complex than conventional consensus protocols for power sharing as developed in [32]. As it is investigated in the simulation/discussion section, failure in LBWCL or master VSI do not affect the stability of MG.

The bode diagram of the open loop transfer function of the proposed method is given in Fig. 6. It is illustrated that the proposed method improves stability margins of the $f-P$ control loop.

\section{NONLINEAR STABILITY ANALYSIS}

First let us obtain an appropriate differential equation describing the proposed $f-P$ control loop. The updated version of droop rule, considering the virtual damping and delta feedback loops, is given as:

$$
\begin{aligned}
& \omega=\omega_{0}-m\left(\frac{\omega_{c}}{s+\omega_{c}}\right)\left(P+D_{v} \Delta \omega\right)-\frac{k}{s}\left(\omega-\omega_{0}\right) \\
& \Rightarrow \Delta \omega=-m\left(\frac{\omega_{c}}{s+\omega_{c}}\right)\left(P+D_{v} \Delta \omega\right)-\frac{k}{s}(\Delta \omega)
\end{aligned}
$$

Noting that $\delta=\Delta \omega / s$ and substituting (3) into (28) with some modification results:

$$
\ddot{\delta}+\left(k+\omega_{c}+m \omega_{c} D_{v}\right) \dot{\delta}+\omega_{c} k \delta+m \omega_{c} A \sin (\delta)=0
$$

where $A=V_{i} V_{j} / X_{i j}$

Equation (29) is a nonlinear second-order equation representing the $f-P$ droop control loop with phase angle feedback. The small signal stability analysis proves the system stability at an operating point at which the linear model is obtained. However, islanded MGs are imposed to large disturbances because of lack of sufficient spinning reserve. So large signal stability analysis is necessary to ensure the system stability at a wide range of operating points. The state variables of the second order system in (29) are defined as:

$$
\begin{aligned}
& x_{1}=\delta \\
& x_{2}=\dot{\delta}=\Delta \omega
\end{aligned}
$$

So the related differential equations are developed as:

-

$x_{1}=x_{2}$

$\dot{x}_{2}=-\left(k+\omega_{c}+m \omega_{c} D_{v}\right) x_{2}-\omega_{c} k x_{1}-m \omega_{c} A \sin \left(x_{1}\right)$

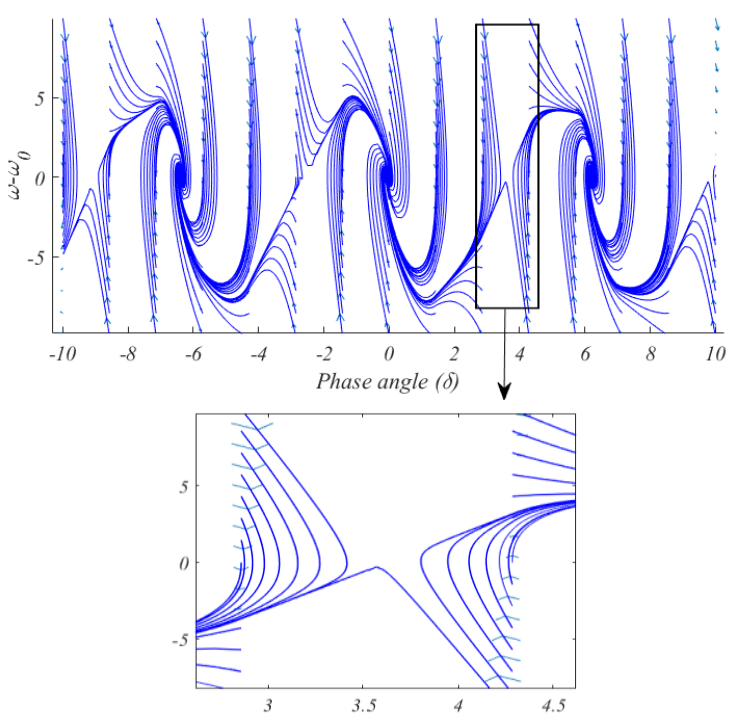

(a)

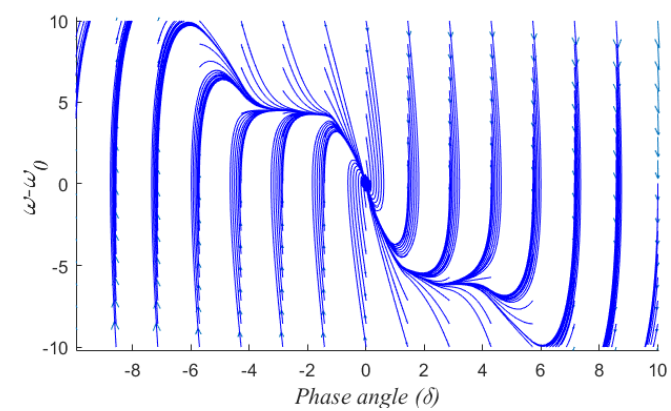

(b)

Fig. 7. Phase portrait: (a) conventional droop $(k=0)$; (b) with feedback loop $(k=1)$.

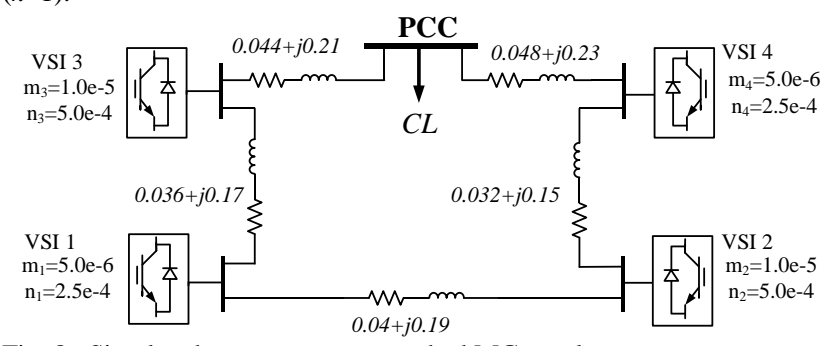

Fig. 8. Simulated autonomous networked MG topology.

The phase portraits (family of trajectories) of a conventional droop control $(k=0)$ and of the proposed method with phase angle feedback are represented in Fig. 7. Fig. 7(a) shows the equilibirium points of conventional droop control which are stable nodes repeated priodically at $(0,0),(2 \pi, 0)$ and $(-2 \pi, 0)$. While the equilibirium points located at $(\pi, 0),(-\pi, 0)$ are saddle points in which trajectories along with stable eigenvector are stable and those along with unstable eigenvector are unstable. Large disturbance in islanded MGs with weak power network (low X/R ratio) may put the MG in this unstable operating region [42].

On the other hand, Fig. 7(b) discloses the phase portrait of the proposed $f$ - $P$ droop control with phase angle feedback loop. The only equilibrium point is $(0,0)$ which is the asymptotically stable node. Accordingly, the phase angle feedback loop improves the stability margins of the $f-P$ control loop. 


\section{Simulation Results}

\section{A. The MG under Study and Simulation Scenario}

An MG including four DG units is simulated in Matlab/Simulink, Simscape toolbox to assess effectiveness of the method. In the simulated MG, which is shown in Fig. 8, all DG units are considered as dispatchable ones (VSIs). Solar cell or wind turbine, with installed storage unit at DC bus, can be regarded as a dispatchable unit which is controlled as a droop-controlled VSI. On the other hand,

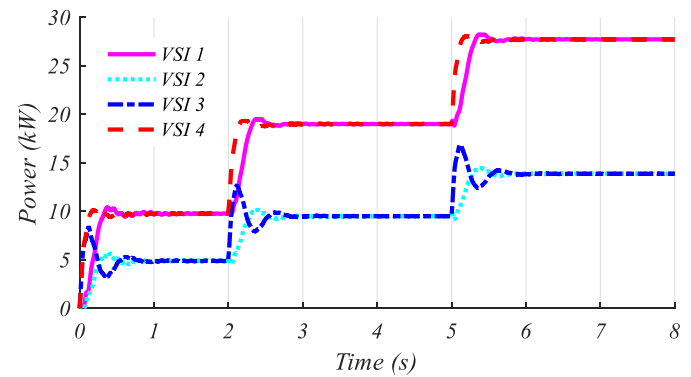

(a)

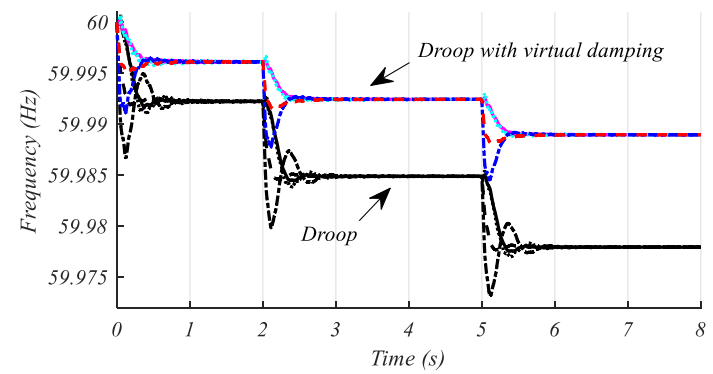

(b)
CSIs (non-dispatchable DG units) follow local voltage variables (magnitude and frequency) of the MG to produce predefined power with fast dynamic [3] and can be considered as negative load. Hence, CSI's dynamic does not affect the MG's voltage and frequency dynamics dominated by droop loops at VSIs and can be ignored. So, the proposed method is applicable to MGs including dispatchable and non-dispatchable DG units since the presence of CSIs does not affect the performance of the method.

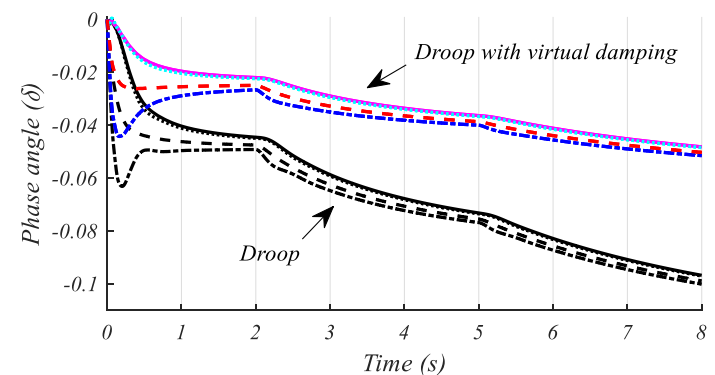

(c)

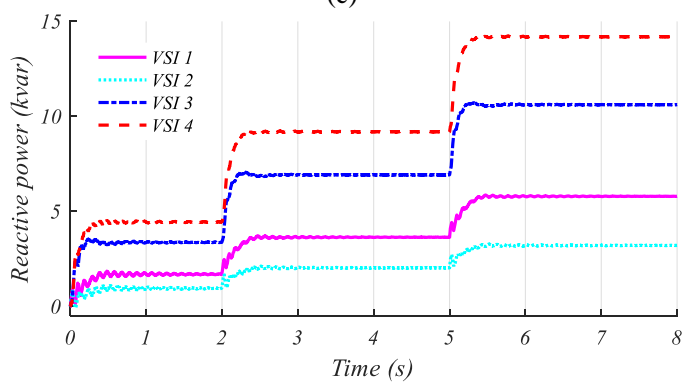

(d)

Fig. 9. Conventional $f-P$ droop controller: (a) power sharing; (b) frequency; (c) phase angle; (d) reactive power.

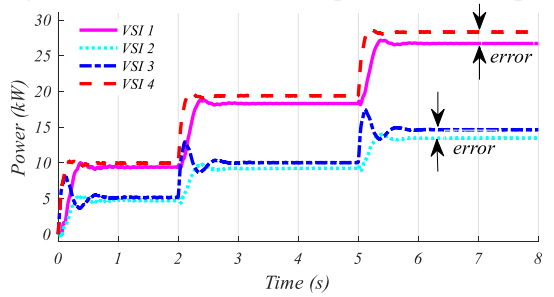

(a)

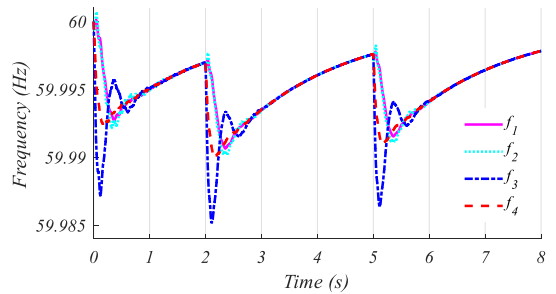

(b)

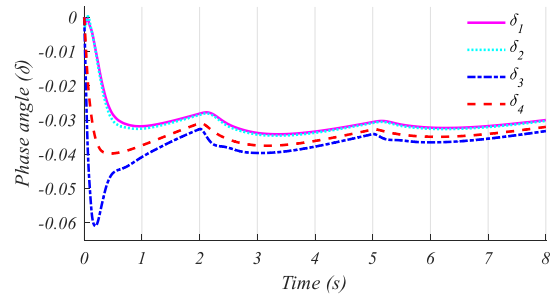

(c)

Fig. 10. Droop controller with conventional frequency restoration: (a) power sharing; (b) frequency; (c) Phase angle.

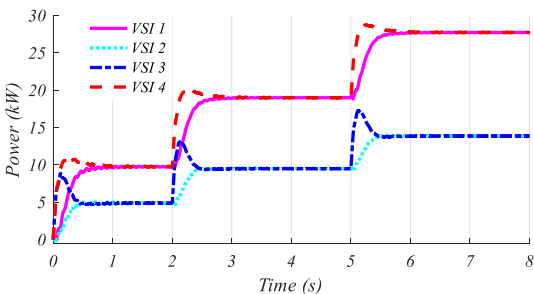

(a)

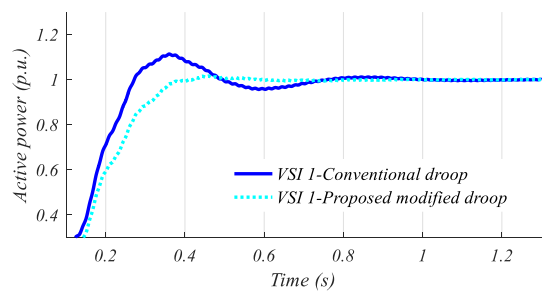

(b)

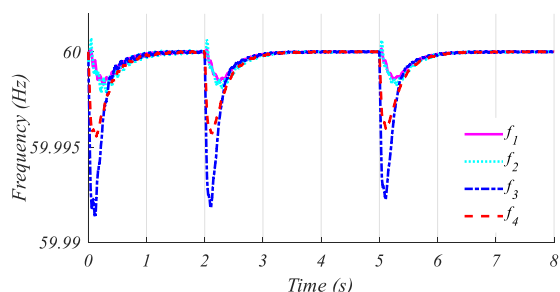

(c)

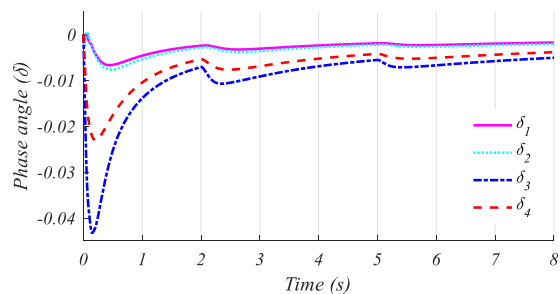

(d)

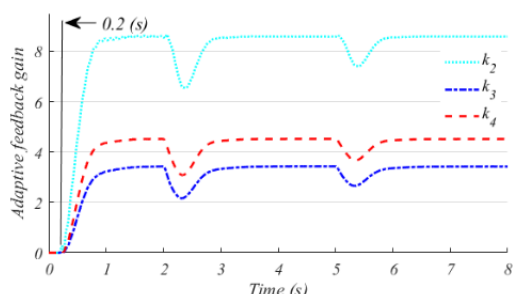

(e)

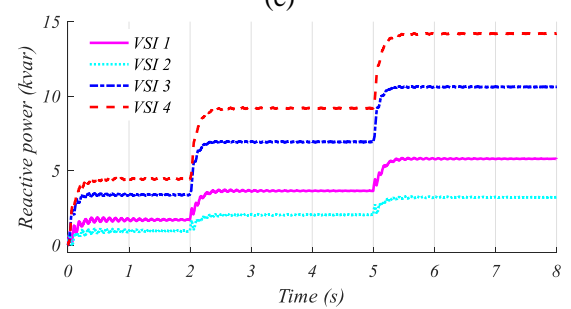

(f)

Fig. 11. Proposed method for frequency restoration: (a) power sharing; (b) dynamic performance; (c) frequency restoration; (d) Phase angle; (e) feedback control loop gain; (f) reactive power. 
In this case study, a common load (CL) $(\mathrm{P}=20 \mathrm{~kW}$ and $\mathrm{Q}=10$ kvar) is connected to the autonomous MG at $\mathrm{t}=0(\mathrm{~s})$. Then the same load is added at $\mathrm{t}=2$ ( $\mathrm{s}$ ) and $\mathrm{t}=5$ ( $\mathrm{s}$ ) to analyze the frequency dynamic when a load change happens as a disturbance. The droop gains of VSI 1 and VSI $4\left(m_{1,4}\right.$ and $n_{1}$, 4) are assumed to be half of those of VSI 2 and VSI $3\left(m_{2,3}\right.$ and $\mathrm{n}_{2,3}$ ). It means that the output power of the former ones must be twice of the output power of latter ones to accurately achieve the power sharing.

In the next subsection, firstly, the drawback of conventional droop control in power sharing, i.e. the frequency deviation, is illustrated and effectiveness of the proposed virtual damping in limiting frequency fluctuation is observed. Then the major problem of conventional frequency restoration methods in frequency restoration, that is inaccuracy of active power sharing, is demonstrated. After that, the performance of the proposed method in frequency restoration, while securing power sharing and dynamic stability, is analyzed.

\section{B. Results}

1) Conventional droop: the performance of the conventional $f$-P droop control in power sharing is illustrated in Fig. 9. Despite of the fact that power sharing is implemented accurately, the frequency deviates from its nominal value, which is the drawback of the droop controller. The frequency deviation, if not restored immediately, would be high after a few disturbances. Nonetheless, the proposed virtual damping is effective to bound frequency deviation as shown in Figs. 9(b). The proposed virtual damping significantly limits frequency fluctuations in comparison to the conventional droop control. It also shortens the time constant of frequency dynamics considerably, almost half that of the conventional droop control, which is consistent with (13). As a result, the virtual damping helps to restore frequency variation immediately. Fig. 9(c) compares the phase angles of generation buses with the droop control loop and with the modified droop loop including a virtual damping. Although the frequency is identical at all DG units, there are different phase angles at MG nodes. It is revealed that the virtual damping improves stability of the droop control as the larger the phase angles, the smaller the stability margin. Fig. 9 (d) reveals the inadequacy in reactive power sharing of the droop control method that can be recovered by a supplementary control loop [13].

2) Conventional frequency restoration: the performance of the conventional frequency restoration loop, which is extensively adopted in the literatures [28]-[30], [33]-[38], is shown in Fig. 10. The frequency restoration loop inserts an error to the power sharing (the error means that the demanded power is not distributed among VSIs according to droop gains so $\mathrm{P}_{1} \neq \mathrm{P}_{4}$ and $\mathrm{P}_{2} \neq \mathrm{P}_{3}$ ). The error in power sharing becomes larger after each disturbance. As it is shown in Fig. 10(b), the response speed is slow and adopting larger control parameter, related to the frequency restoration loop, for faster response leads to a larger error and more inaccuracy in the active power sharing.

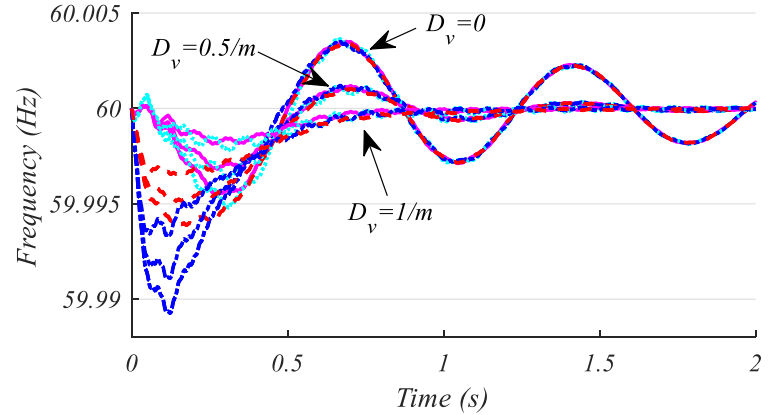

Fig. 12. The virtual damping impact on compensating time delay imposed by communication link.

3) Proposed method: the performance of the proposed method is depicted in Fig. 11. The power sharing is implemented accurately with improved dynamic (damped fluctuations), as shown in Fig. 11(a). Fig. 11(b) reveals the improvement in the dynamic performance of the proposed modified droop loop in comparison to the conventional droop control. Fig. 11(c) indicates a significant improvement in the frequency restoration via the proposed method in terms of quantity (amount of frequency deviations) and quality (time constant of frequency restoration) in comparison to the results shown in Fig. 9(b) and Fig. 10(b), and particularly it is consistent with the so-called precise power sharing. The feedback loop gain corresponding to VSI 1, as master one, is fixed to $k_{1}=10$ and the feedback gains of VSIs 2-4 are determined adaptively by the proposed method. The time delay related to transferring the data through the communication link is considered as $200 \mathrm{~ms}$ (as a worst case), which can be monitored in Fig. 11(e). Comparing Fig 9(d) with Fig. 11(f) shows that the reactive power sharing is not affected by the method.

\section{DISCUSSION}

As per the theoretical analysis and simulation results presented in this work, the proposed method bounds the frequency fluctuations, restores the frequency deviations and improves dynamic stability margin of the autonomous networked MG, while securing precise active power sharing. The low frequency oscillations related to conventional $f-P$ droop loop are also damped with the adapted droop control, as shown in Fig. 11(b). In addition, since the frequency is kept close to the nominal value and phase angle variation is restricted, the plug-and-play capability of DG units as well as synchronizing with the upstream grid is more straightforward. The proposed method requires an LBWCL to preserve accurate power sharing for frequency restoration. Nevertheless, the performance improvement justifies the additional communication requirement considering the following details:

1. since only a dc value is distributed to DG units (the frequency of phase angle dynamics is very low), the existing LBWCL is sufficient without imposing extra costs;

2. the transmission delay $\left(D_{T}\right)$ (data-rate) of the communication link depends on the size (bits) of transferred data and has nothing to do with the distance in a local area like MG. Note that $D_{T}=N / R(s)$ where $N$ is 
the number of bits and $R$ is the rate of transmission (bit/s). For instance, for transferring a dc value $(8 \mathrm{bits})$ through a communication link with a data rate of $1 \mathrm{kbit} / \mathrm{s}$, the transmission delay is $8 \mathrm{~ms}$.

3. from (29) the time constant of the proposed control loop is $T^{\prime \prime}{ }_{d}=2 /\left(k+\omega_{c}+m \omega_{c} D_{v}\right)$, which is $50 \mathrm{~ms}$ for $\omega_{c}=15 \mathrm{rad} / \mathrm{s}$ and $k=10$. The phase angle variation reaches to a final value after $5 T^{\prime \prime}{ }_{d}=250 \mathrm{~ms}$. So $D_{T}$ up to $4 T^{\prime \prime}{ }_{d}=200 \mathrm{~ms}$ does not significantly influence the system performance. So $k$ is selected considering the communication delay of the existing LBWCL. Nevertheless, the communication delay $D_{T}=200 \mathrm{~ms}$ is taken as the worst-case scenario and the actual delay is much less due to small size of the transmitted data.

4. the proposed virtual damping critically helps to compensate the communication delay as shown in Fig. 12. The delayed restoration signal (due to transmission delay through the communication link) is high which leads to frequency fluctuations even in steady state. Since the virtual damping limits the frequency deviation, the phase angle variation and consequently the delayed restoration signal are also limited, which prevents the frequency from fluctuating in steady state.

5. all frequency restoration loops must work simultaneously to avoid inaccuracy in active power sharing. However, the time delay of communication link is an issue. In order to take the time delay of communication link into account, a delay equal to $D_{T}$ must be applied to the frequency restoration loop at the master VSI. To this end, the control signal of master VSI is taken from the local communication link.

6. failure in the communication link or in the master VSI does not threat the stability of $\mathrm{MG}$, since only the frequency restoration loop is lost $\left(k_{i}=0\right)$ and active power sharing and virtual damping are still precisely implemented. The outage of master VSI can be detected and replaced with an abundant VSI by the MGCC.

\section{CONCLUSION}

This paper considers droop control performance in active power sharing among DG units in MGs. Drawing a new analogy between droop control in conventional synchronous generators and droop control in MGs illustrates that low-pass filter provides virtual inertia in converter-based MGs. However, lack of damping factor in droop-like control in MGs makes the frequency deviation high. To address the issue, virtual damping as well as corresponding selection criteria are proposed to reduce the frequency fluctuation in MGs. It is also investigated that the conventional frequency restoration method is in conflict with accurate power sharing. Hence, a novel method, based on phase angle feedback, is developed to overcome the drawback of conventional methods in frequency restoration including a control system to tune the corresponding feedback gains adaptively. The phase portrait of the second-order non-linear equation describing the proposed $f$ - $P$ loop reveals that the proposed method is stable in a variety of operating points. Simulating an MG consisting of four DG units connected to a meshed power network confirms the validity of the statements and proves the effectiveness of the proposed method.

Although the controllers at individual DG units are synchronized through a communication signal, it has been verified that a failure or delay in the communication link do not affect the stability of the MG. The proposed method is fast, simple and effective, while preserving plug-and-play capability to remove errors caused during implementing the controller whenever a new generation unit is included.

The advantage of the method over consensus-based methods presented in [32]-[38] is that the frequency restoration is not in conflict with power sharing, while the stability of the $f$ - $P$ control loop is guaranteed. In addition, the required bandwidth is lower because of one directional flowing and smaller size of data. The superiority of the control technique over the phase angle droop methods [26] is due to the GPS failure in time synchronization that leads to inaccurate power sharing in $\delta$ - $P$ control methods and to MG collapse, while in the proposed method GPS timing is not necessary. Moreover, frequency restoration is completely implemented by the proposed method.

\section{REFERENCES}

[1] M. H. Moradi and M. Eskandari, "A hybrid method for simultaneous optimization of DG capacity and operational strategy in microgrids considering uncertainty in electricity price forecasting," Renewable Energy, vol. 68, pp. 697-714, 2014.

[2] M. H. Moradi, M. Eskandari, and S. M. Hosseinian, "Operational strategy optimization in an optimal sized smart microgrid," IEEE Trans. on Smart Grid, vol. 6, no. 3, pp. 1087-1095, 2015.

[3] M. H. Moradi, M. Eskandari, and S. M. Hosseinian, "Cooperative control strategy of energy storage systems and micro sources for stabilizing microgrids in different operation modes," International Journal of Electrical Power \& Energy Systems, vol. 78, pp. 390-400, 2016.

[4] J. Rocabert, A. Luna, F. Blaabjerg, and P. Rodriguez, "Control of power converters in AC microgrids," IEEE Trans. on Power Electron., vol. 27, no. 11, pp. 4734-4749, 2012.

[5] M. C. Chandorkar, D. M. Divan, and R. Adapa, "Control of parallel connected inverters in standalone AC supply systems," IEEE Trans. on Ind. Appl., vol. 29, no. 1, pp. 136-143, 1993.

[6] H. Han, X. Hou, J. Yang, J. Wu, M. Su, and J. M. Guerrero, "Review of power sharing control strategies for islanding operation of AC microgrids," IEEE Trans. on Smart Grid, vol. 7, no. 1, pp. 200-215, 2016.

[7] Y. Han, H. Li, P. Shen, E. A. A. Coelho, and J. M. Guerrero, "Review of active and reactive power sharing strategies in hierarchical controlled microgrids," IEEE Trans. on Power Electron., vol. 32, no. 3, pp. 24272451, 2017.

[8] J. M. Guerrero, L. G. De Vicuna, J. Matas, M. Castilla, and J. Miret, "Output impedance design of parallel-connected UPS inverters with wireless load-sharing control," IEEE Trans. on Ind. Electron., vol. 52, no. 4, pp. 1126-1135, 2005.

[9] C. K. Sao and P. W. Lehn, "Autonomous load sharing of voltage source converters," IEEE Trans. on Power Del., vol. 20, no. 2, pp. 1009-1016, 2005.

[10] He, Jinwei, and Yun Wei Li. "An enhanced microgrid load demand sharing strategy." IEEE Trans. on Power Electron., vol. 27, no. 9, 3984$3995,2012$.

[11] J. He, Y. W. Li, J. M. Guerrero, F. Blaabjerg, and J. C. Vasquez, "An Islanding Microgrid Power Sharing Approach Using Enhanced Virtual Impedance Control Scheme," IEEE Trans. on Power Electron., vol. 28, no. 11, pp. 5272-5282, Nov. 2013.

[12] Y. Zhu, F. Zhuo, F. Wang, B. Liu, R. Gou, Y. Zhao, “A Virtual Impedance Optimization Method for Reactive Power Sharing in Networked Microgrid," IEEE Trans. on Power Electroni., vol. 31, no. 4, pp. 28902904, 2016.

[13] M. Eskandari, Li Li, and M. H. Moradi. "Decentralized Optimal Servo Control System for Implementing Instantaneous Reactive Power Sharing in Microgrids." IEEE Trans. on Sustain. Energy, vol. 9, no. 2, pp. 525537, 2018. 
[14] Shafiee, Qobad, Josep M. Guerrero, and Juan C. Vasquez. "Distributed secondary control for islanded microgrids - A novel approach," IEEE Trans. on Power Electron., vol. 29, no. 2, pp. 1018-1031, 2014.

[15] J. W. Simpson-Porco, Q. Shafiee, F. D”orfler, J. M. Vasquez, J. M. Guerrero, and F. Bullo, "Secondary frequency and voltage control of islanded microgrids via distributed averaging," IEEE Trans. on Industrial Electronics, vol. 62, no. 11, pp. 7025-7038, 2015.

[16] Lin-Yu Lu, Chia-Chi Chu, "Consensus-Based Droop Control Synthesis for Multiple DICs in Isolated Micro-Grids," IEEE Trans. on Power Syst., vol. 30, no. 5, pp. 2243-2256, 2015.

[17] H. Zhang, S. Kim, Q. Sun, J. Zhou, "Distributed Adaptive Virtual Impedance Control for Accurate Reactive Power Sharing Based on Consensus Control in Microgrids," IEEE Trans. on Smart Grids, vol. 6, no. 3, pp. 1087-1095, May. 2016.

[18] J. Schiffer, T. Seel, J. Raisch, T. Sezi, "Voltage Stability and Reactive Power Sharing in Inverter-Based Microgrids with Consensus-Based Distributed Voltage Control," IEEE Trans. on Cont. Syst. Tech.,, vol. 24, no. 10, pp. 96-109, 2016.

[19] Lou, Guannan, Wei Gu, Yinliang Xu, Ming Cheng, and Wei Liu. "Distributed MPC-Based Secondary Voltage Control Scheme for Autonomous Droop-Controlled Microgrids," IEEE Trans. on Sustain. Energy, vol. 8, no. 2, pp. 792-804, 2017.

[20] Zhang, Guoyue, Chaoyong Li, Donglian Qi, and Huanhai Xin. "Distributed Estimation and Secondary Control of Autonomous Microgrid," IEEE Trans. on Power Syst., vol. 32, no. 2, pp. 989-998, 2017.

[21] E. Barklund, N. Pogaku, M. Prodanovic, C. Hernandez-Aramburo, T. C. Green. "Energy management in autonomous microgrid using stabilityconstrained droop control of inverters," IEEE Trans. on Power Electron., vol. 23, no. 5, pp. 2346-2352, 2008.

[22] Y. A. I. Mohamed, E. F. El-Saadany, "Adaptive decentralized droop controller to preserve power sharing stability of paralleled inverters in distributed generation microgrids," IEEE Trans. on Power Electron., vol. 23, no. 6, pp. 2806-2816, 2008.

[23] C. N. Rowe, T. J. Summers, R. E. Betz, J. C. David, G. M. Timothy, "Arctan power-frequency droop for improved microgrid stability," IEEE Trans. on Power Electron., vol. 28, no. 8, pp. 3747-3759, 2013.

[24] Q. C. Zhong, G. Weiss, "Synchronverters: Inverters that mimic synchronous generators," IEEE Trans. on Ind. Electron., vol. 58, no. 4, pp. 1259-1267, 2011.

[25] M. N. Marwali, J. W. Jung, A. Keyhani, "Control of distributed generation systems-Part II: Load sharing control," IEEE Trans. on Power Electron., vol. 19, no. 6, pp. 1551-1561, 2004.

[26] R. Majumder, B. Chaudhuri, A. Ghosh, R. Majumder, G. Ledwich, F. Zare, "Improvement of stability and load sharing in an autonomous microgrid using supplementary droop control loop," IEEE Trans. on Power Syst., vol. 25, no. 2, pp. 796-808, 2010.

[27] M. S. Golsorkhi, D. Lu, Josep M. Guerrero, "A GPS-based decentralized control method for islanded microgrids." IEEE Trans. on Power Electron., vol. 32, no. 2, pp. 1615-1625, 2017.

[28] J. M. Guerrero, J. C. Vasquez, J. Matas, "Hierarchical control of droopcontrolled AC and DC microgrids-A general approach toward standardization," EEE Trans. on Ind. Electron., vol. 58, no. 1, pp. 158172, 2011.

[29] A. Bidram, A. Davoudi, "Hierarchical structure of microgrids control system," IEEE Trans. on Smart Grids, vol. no. 4, pp. 1963-1976, 2012.

[30] F. Katiraei, M. R. Iravani, "Power Management Strategies for a Microgrid With Multiple Distributed Generation Units" IEEE Trans. on Power Sys., vol. 21, no. 4, pp. 1821-1831, 2006.

[31] M. Kosari, S. H. Hosseinian. "Decentralized reactive power sharing and frequency restoration in islanded microgrid," IEEE Trans. on Power Systems, vol. 32, no. 4, pp. 2901-2912, 2017.

[32] F. Guo, C. Wen, J. Mao, Y. D. Song, "Distributed secondary voltage and frequency restoration control of droop-controlled inverter-based microgrids," IEEE Trans. on industrial Electronics, vol. 62, no. 7, pp. 4355-4364, 2015.

[33] S. Zuo, A. Davoudi, Y. Song, F. L. Lewis, "Distributed finite-time voltage and frequency restoration in islanded AC microgrids," EEE Trans. on Ind. Electron., vol. 63, no. 10, 5988-5997, 2016.

[34] C. Ahumada, R. Cárdenas, D. Saez, J. M. Guerrero. "Secondary control strategies for frequency restoration in islanded microgrids with consideration of communication delays," IEEE Trans. on Smart Grid, vol. 7, no. 3, pp. 1430-1441, 2016.
[35] X. Wu, C. Shen, R. Iravani, "A distributed, cooperative frequency and voltage control for microgrids," IEEE Trans. on Smart Grid, vol. 9, no. 4, pp. 2764-2776, 2018.

[36] Y. S. Kim, E. S. Kim, S. I. Moon. "Distributed generation control method for active power sharing and self-frequency recovery in an islanded microgrid," IEEE Trans. on Power Syst., vol. 32, no. 1, pp. 544-551, 2017.

[37] N. M. Dehkordi, N. Sadati, M. Hamzeh. "Fully Distributed Cooperative Secondary Frequency and Voltage Control of Islanded Microgrids," IEEE Trans. on Energy Conversion, vol. 32, no. 2. 675-685, 2017.

[38] A. Pilloni, A. Pisano, E. Usai, "Robust Finite-Time Frequency and Voltage Restoration of Inverter-Based Microgrids via Sliding-Mode Cooperative Control," EEE Trans. on Industrial Electronics, vol. 65, no. 1, 907-917, 2018.

[39] K. Ogata, Y. Yang, "Modern control engineering," Prentice hall India, 2002.

[40] S. Tong and K. N. Miu, "A network-based distributed slack bus model for DGs in unbalanced power flow studies," IEEE Trans. on Power Sys., vol. 20, no. 2, pp. 835-842, 2005.

[41] M. A. Abdelaziz, "Effect of detailed reactive power limit modeling on islanded microgrid power flow analysis," IEEE Trans. on Power Syst., vol. 31, no. 2, pp. 1665-1666, 2016.

[42] M. Eskandari, L. Li, M. H. Moradi, and P. Siano, "A nodal approach based state-space model of droop-based autonomous networked microgrids," Sustain. Energy, Grids and Networks, pp. 100216, 2019.

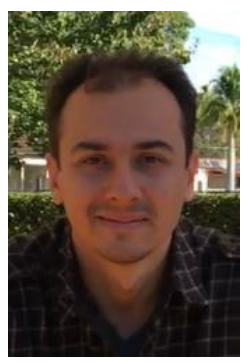

Mohsen Eskandari was born in Saveh, Iran. He received the B.Sc. and M.Sc. degrees in electrical engineering from Islamic Azad University, Saveh branch, Saveh, Iran, in 2004 and 2013, respectively. Currently, he is pursuing the Ph.D. degree in electrical engineering at University of Technology Sydney (UTS), Sydney, Australia, since Jan 2017.

His current research interests include power systems modelling \& dynamics, control, optimization, artificial intelligence, and power electronics, particularly their applications into Smart/Micro Grids. He has more than 10 years of experience in different parts of the electrical industry. He has proven skills handling power systems construction projects as well as a strong background in the field of automation and control (PLC). He also possesses valuable experience in relation to power systems in transmission, distribution, and load (industrial and residential) levels.

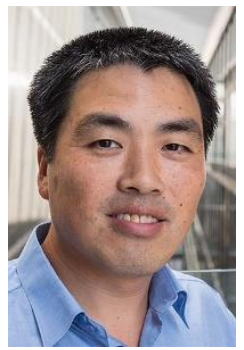

Li Li (S'04-M'11) received his B.S. degree from Huazhong University of Science and Technology in 1996, M.S. degree from Tsinghua University in 1999, and Ph.D. degree from University of California, Los Angeles in 2005 respectively, all in Electrical Engineering. From 2005 to 2007 he was a research associate at the University of New South Wales at the Australian Defence Force Academy (UNSW@ ADFA). From 2007 to 2011, he was a researcher at the National ICT Australia, Victoria Research Laboratory, Department of Electrical and Electronic Engineering, The University of Melbourne. He joined University of Technology Sydney in 2011 and currently he is an Associate Professor. Dr Li held several visiting positions at various universities. His research interests are control theory and power system control. He is presently serving as an Associate Editor of IET Renewable Power Generation, IET Generation, Distribution and Transmission, and IET Smart Grid.

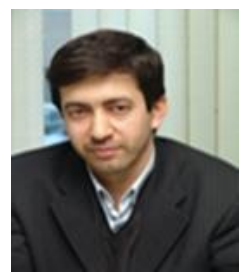

Mohammad Hassan Moradi is a full professor in the department of electrical engineering, Bu-Ali Sina University, Hamedan, Iran. He obtained his B.Sc., M.Sc. and Ph.D. degrees from Sharif University of Technology, Tarbiat Modares University and Strathclyde University in Glasgow, Scotland in 1991, 1993, and 2002, respectively. His research interests include New and Green Energy, Micro Grid Modeling and Control, DG Location and Sizing in Power Systems, Photovoltaic Systems and Power Electronics, Combined Heat and Power Plant, Power Quality, Supervisory Control, and Fuzzy Control. http://profs.basu.ac.ir/mh-moradi/ 


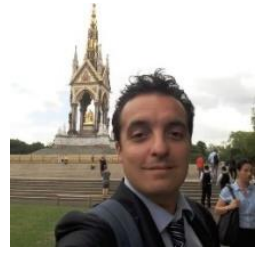

Pierluigi Siano (M'09-SM'14) received the M.Sc. degree in electronic engineering and the Ph.D. degree in information and electrical engineering from the University of Salerno, Salerno, Italy, in 2001 and 2006, respectively.

$\mathrm{He}$ is a Professor and Scientific Director of the Smart Grids and Smart Cities Laboratory with the Department of Management \& Innovation Systems,

University of Salerno.

His research activities are centered on demand response, on the integration of distributed energy resources in smart grids and on planning and management of power systems.

He has co-authored more than 420 papers including more than 200 international journal papers that received more than 9000 citations with an $\mathrm{H}$-index equal to 46. He has been the Chair of the IES TC on Smart Grids.

$\mathrm{He}$ is an Associate Editor of the IEEE TRANSACTIONS ON INDUSTRIAL INFORMATICS, of the IEEE TRANSACTIONS ON INDUSTRIAL ELECTRONICS and of IET Renewable Power Generation.

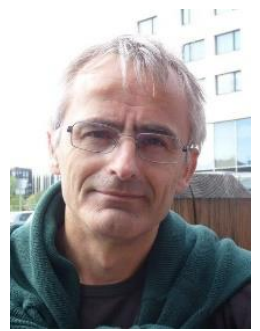

Frede Blaabjerg (S'86-M'88-SM'97-F'03) was with ABB-Scandia, Randers, Denmark, from 1987 to 1988. From 1988 to 1992, he got the PhD degree in Electrical Engineering at Aalborg University in 1995. He became an Assistant Professor in 1992, an Associate Professor in 1996, and a Full Professor of power electronics and drives in 1998. From 2017 he became a Villum Investigator. $\mathrm{He}$ is honoris causa at University Politehnica Timisoara (UPT), Romania and Tallinn Technical University (TTU) in Estonia.

His current research interests include power electronics and its applications such as in wind turbines, PV systems, reliability, harmonics and adjustable speed drives. He has published more than 600 journal papers in the fields of power electronics and its applications. He is the co-author of four monographs and editor of ten books in power electronics and its applications.

He has received 30 IEEE Prize Paper Awards, the IEEE PELS Distinguished Service Award in 2009, the EPE-PEMC Council Award in 2010, the IEEE William E. Newell Power Electronics Award 2014, the Villum Kann Rasmussen Research Award 2014 and the Global Energy Prize in 2019. He was the Editor-in-Chief of the IEEE TRANSACTIONS ON POWER ELECTRONICS from 2006 to 2012. He has been Distinguished Lecturer for the IEEE Power Electronics Society from 2005 to 2007 and for the IEEE Industry Applications Society from 2010 to 2011 as well as 2017 to 2018. In 2019-2020 he serves as President of IEEE Power Electronics Society. He is Vice-President of the Danish Academy of Technical Sciences too.

$\mathrm{He}$ is nominated in 2014-2018 by Thomson Reuters to be between the most 250 cited researchers in Engineering in the world. 